\title{
Endovascular repair of traumatic rupture of the aortic isthmus: Midterm results
}

\author{
Bertrand Marcheix, MD, ${ }^{a}$ Camille Dambrin, MD, PhD, ${ }^{a}$ Jean-Philippe Bolduc, MD, ${ }^{b}$ Catherine Arnaud, MD, ${ }^{\mathrm{c}}$ \\ Lucy Hollington, MD, ${ }^{d}$ Christophe Cron, MD, ${ }^{a}$ Antoine Mugniot, MD, ${ }^{a}$ Philippe Soula, MD, ${ }^{a}$ Mehdi Bennaceur, MD, ${ }^{b}$ \\ Valérie Chabbert, MD, ${ }^{\mathrm{b}}$ Philippe Otal, MD, ${ }^{\mathrm{b}}$ Alain Cérène, $\mathrm{MD}^{\mathrm{a}}{ }^{\mathrm{a}}$ and Hervé Rousseau, MD, $\mathrm{PhD}^{\mathrm{b}}$
}

Objective: The endovascular management of aortic traumatic ruptures has been proposed as an alternative to classical surgical procedures. The aim of this work was to report the midterm results of the endovascular treatment of traumatic ruptures of the isthmic aorta.

Methods: Between January 1996 and July 2005, endovascular repair of blunt traumatic aortic ruptures was performed in 33 patients (mean age, $40 \pm 17$ years). The stent grafts used were either Talent Medtronic $(\mathrm{n}=27)$, Gore Excluder $(\mathrm{n}=$ $4)$, or Boston Vanguard $(\mathrm{n}=2)$ grafts. Follow-up was $94.9 \%$ complete and averaged $32.4 \pm 28.8$ months (maximum, 8 years).

Results: Stent graft deployment was successful in all cases without need for surgical conversion. Except for one iliac rupture, which was treated with an iliofemoral bypass during the same procedure, there was no major perioperative complication. The early complications consisted of 3 primary endoleaks ( 1 type I and 2 type IV), 1 transient paraparesis, 1 occlusion of the main left bronchus, 1 thrombosis, and 2 pseudoaneurysms of the brachial artery. All the primary endoleaks healed within the first month. No patient died, and no aortic reinterventions were performed. The midterm complications were a mild circumferential thrombus at the distal part of the stent graft and a fracture of the nitinol stent. Both complications were asymptomatic and were discovered on systematic computed tomographic scan examination. Actuarial freedom from complication at 1 year was $96.1 \% \pm 3.8 \%$ and $85.5 \% \pm 10.6 \%$ at 3 and 5 years, respectively.

Conclusion: This study demonstrates that the endovascular treatment of blunt thoracic aortic traumatisms is a safe and effective therapeutic method without increased midterm morbidity and mortality rates.

From the Departments of Thoracic and Cardiovascular Surgery ${ }^{\mathrm{a}}$ and Radiology, ${ }^{\mathrm{b}}$ Rangueil University Hospital, Toulouse, France; the Department of Epidemiology and Biostatistics, ${ }^{c}$ University Hospital, Toulouse, France; and the Department of Cardiology, ${ }^{\mathrm{d}}$ Montauban General Hospital, Montauban, France.

Address for reprints: Hervé Rousseau, MD, Radiology Department, Rangueil University Hospital, TSA 50032, 1 avenue Jean Poulhès, 31059 Toulouse, Cedex 9, France (E-mail: rousseau.h@chu-toulouse.fr).

J Thorac Cardiovasc Surg 2006;132:1037-41 $0022-5223 / \$ 32.00$

Copyright $\odot 2006$ by The American Association for Thoracic Surgery

doi:10.1016/j.jtcvs.2006.07.004
$\mathrm{U}$ sually, blunt thoracic aortic ruptures occur at the level of the aortic isthmus when deceleration or thoracic crushing mechanisms are involved. ${ }^{1}$ Eighty percent of the victims die at the scene of the accident. Aortic disruption is most of the time associated with other life-threatening injuries. This makes treatment of the survivors extremely challenging. In spite of technical advances in aortic surgery, the surgical treatment of those who survive such an accident remains controversial, and the mortality and morbidity rates remain high. For the most part, patients' deaths are linked with the associated injuries, which might in fact have been worsened by the operation and the circulatory assistance technique. ${ }^{2-4}$ Nevertheless, the use of extracorporeal circulation is the only technique that enables a significant reduction in the rate of paraplegia. Postoperative paraplegia is the main neurological complication, with a rate of occurrence ranging between $3 \%$ and $20 \%$ in the most experienced trauma centers. ${ }^{1,3,4}$ Delayed surgical treatment with circulatory assistance has been advocated as being a good compromise. However, the 


\section{Abbreviations and Acronyms \\ $\mathrm{CT}=$ computed tomography \\ LSCA $=$ left subclavian artery}

choice of having a period of waiting is not free of risk. ${ }^{1,4,5}$ Under these circumstances, endovascular repair appears to be a potential therapeutic alternative.

In 1999, our group published the short-term results of using this technique. ${ }^{6}$ Today, more than 300 cases of endovascular repair of aortic ruptures have been reported, either as small series or as case reports. All had only a very limited follow-up period.

The aim of this work is to report the midterm results of the endovascular treatment of blunt traumatic ruptures of the aortic isthmus.

\section{Patients and Methods Patients}

Between January 1996 and July 2005, 33 patients with traumatic pseudoaneurysms of the aortic isthmus underwent endovascular repair and were included in a prospective, monocentric, nonrandomized study. The use of stent grafts for this particular medical condition was approved by our ethical committee. Informed consent was obtained in all cases, either from the patients themselves or from their relatives. There were 29 male patients with a mean age of 35.8 \pm 17.8 years (range, 16-75 years) at the time of the surgical procedure, and there were 4 female patients with a mean age of 55.6 \pm 9.8 years (range, 39-63 years) at the time of the surgical procedure.

Initially, endovascular management was only performed after the patient had recovered from any associated life-threatening injuries. More recently, stent grafts of different sizes have become readily available in our department, making it possible to perform endovascular procedures even in emergency situations.

Twenty-one patients were treated between 14 and 252 days after the traumatic injury had been sustained (subacute settings), with an average of $93.6 \pm 83.2$ days. Twelve patients were treated between day 0 and day 14 after the trauma (acute settings), with a mean of $6.2 \pm 4.5$ days. Four of the latter 12 patients were treated within the first 24 hours after the accident.

\section{Associated Injuries}

The mean Injury Severity Score was $40.2 \pm 10.7$ (range, 13-57). All patients who were treated in acute or subacute settings had been judged to have contraindications for conventional surgical interventions involving circulatory assistance because of their associated injuries. Twenty-nine (87.8\%) patients presented with severe encephalic injuries. Thirty-three (100\%) patients presented with severe thoracic traumatisms with lung contusion or contralateral flail chest. Twenty-six (78.8\%) patients presented with major fractures, and 18 (54.5\%) patients presented with intra-abdominal lacerations.

\section{Preoperative Morphologic Assessment}

Currently, a multidetectors computed tomographic (CT) scan with multiplanar reconstruction is performed so as to precisely describe the aortic injury and assess the iliofemoral access. All the pseudoaneurysms we treated were localized at the level of the isthmic aorta. They had a mean diameter of $37.1 \pm 10.6 \mathrm{~mm}$ (range, $25-70$ $\mathrm{mm})$. The mean proximal aortic neck diameter was $25.9 \pm 3.2 \mathrm{~mm}$ (range, 21-30.5 mm). The mean distance between the lesion and the ostium of the left subclavian artery (LSCA) was $17.9 \pm 13.7$ $\mathrm{mm}$ (range, 0-55 mm). The diameter of the iliofemoral junction was at least $6 \mathrm{~mm}$ in all cases.

\section{Intravascular Devices}

Four Excluder (W. L. Gore \& Associates, Elkton, MD) devices, 27 Talent (Medtronic, Inc, Minneapolis, Minn) devices, and 2 Vanguard (Boston Scientific, Natick, Mass) devices were used. The stent grafts were about $10 \%$ to $15 \%$ oversized compared with the diameter of the normal aorta. They were also at least 30 to $40 \mathrm{~mm}$ longer than the lesion to be treated. The mean diameter of the stent grafts was $28.4 \pm 4.1 \mathrm{~mm}$ (range, $24-40 \mathrm{~mm}$ ), the mean length was $111.8 \pm 17.4 \mathrm{~mm}$, and the mean covered length was $97.2 \pm 13.6$ mm (range, 70-130 mm).

\section{Endovascular Procedures, Postoperative Treatment, and Follow-up of Patients}

All the procedures were carried out in the operating theater suite of the interventional radiology department. The procedures themselves involved a multidisciplinary team composed of interventional radiologists, cardiovascular surgeons, cardiologists, and anesthesiologists.

The endovascular technique used was the same as previously described. ${ }^{6}$ A large-spectrum prophylactic antibiotic (cefuroxime, $1500 \mathrm{mg}$ administered intravenously die) was systematically administered during the procedure and maintained for 24 hours after the procedure. The long-term postoperative treatment consisted of aspirin ( $325 \mathrm{mg}$ daily). In cases involving invasive procedures, the recommendations given to prevent infection of the devices were the same as those given to patients with heart valve prostheses. The follow-up consisted in carrying out transesophageal echocardiography, CT scans, or magnetic resonance before discharge and then at the 3rd, 6th, 12th, and 18th months. CT scanning or magnetic resonance was then performed yearly. Two patients who lived at a considerable distance from our hospital refused to have radiologic follow-up and were thus considered lost from follow-up (5.12\%). The mean follow-up duration for the others was $2.7 \pm 2.4$ years (maximum, 8 years). Ten patients had a follow-up lasting 5 years or more.

\section{Definitions}

The definitions retained in this study are described in the literature. ${ }^{7}$ Primary technical success was defined as a complete exclusion of the false aneurysm without the necessity of any additional procedure and without perioperative death or complication. Secondary technical success was defined as a complete exclusion of the false aneurysm after an additional endovascular procedure or after spontaneous thrombosis of primary endoleak within the first month. Endoleak was defined as blood flow apart from the stent graft. Endoleaks were then classified according to the literature. ${ }^{8,9}$ 
Any intraoperative or perioperative death, any primary or secondary endoleak, any failure of the endovascular device, any reintervention, any death related to an aortic rupture, or any sudden unexplained late death were considered failures of the treatment.

\section{Statistical Analysis}

The continuous variables were expressed as means \pm standard deviation. The evaluation of actuarial survivals was carried out according to the Kaplan-Meier nonparametric method. The actuarial survivals are reported at 1,3, and 5 years (with 95\% confidence intervals). The analysis was performed with the STATA program (Stata Corp, College Station, Tex).

\section{Results \\ Procedures}

An infrainguinal iliofemoral access was performed for all the patients, and a single stent graft was used in all cases. The mean duration of the procedures was $105.0 \pm 47.1$ minutes (range, 60-210 minutes). Twenty-nine patients received an intravenous heparin injection in the amount of 75 UI/kg. Four (12.2\%) patients did not receive heparin because of their severe hemorrhagic encephalic injuries, and none of them had any subsequent complications requiring reintervention with heparin administration. The ostium of the LSCA was covered in 9 (27.3\%) patients. The noncovered proximal part of the stent graft was positioned at the level of the ostium of the LSCA in $23(69.7 \%)$ patients.

\section{Immediate Results}

Complete exclusion of the false aneurysm was obtained in 30 patients. The primary success rate was $90.9 \%$. Three patients presented with a mild endoleak at the end of the procedure on angiographic control. These primary endoleaks were also identified on the first CT scan, which was performed 24 or 48 hours after the procedure. One was a proximal type I endoleak, and 2 were type IV endoleaks (Talent; Medtronic, Inc). These 3 primary endoleaks underwent thrombosis, and they became undetectable on the CT scan after 1 month. No aortic reinterventions and no additional endovascular procedures were carried out. No perioperative deaths occurred. One (3\%) patient presented with a rupture of the external iliac artery. The hemorrhage was controlled with an aortic occlusive balloon at the level of the infrarenal aorta. The arterial rupture was treated with an iliofemoral bypass, which was performed at the time of the procedure.

\section{Early Postoperative Course, Endoleaks, and Early Reinterventions ( $<30$ Days)}

Five $(15.5 \%)$ early postoperative complications were observed. One patient (whose LSCA had been covered) presented with a transient paraparesis (day 0), which was resolved in 3 days after medical treatment had been given, including cerebrospinal fluid drainage. One patient presented with an acute ischemia of the left upper limb (day 0) because of a thrombosis of the brachial artery at the level of the introducer requiring an embolectomy. Two patients presented with a false aneurysm of the left brachial artery (day 1). They underwent surgical intervention. One patient presented with an atelectasis of the left main bronchus (day 4) requiring the placement of an endobronchial silicone prosthesis for 3 months. Mean shrinkage during the first month was $11.5 \pm 7.9 \mathrm{~mm}$. Only $3(9.1 \%)$ patients presented with a transient progression of the size of the false aneurysm. After 6 months, all the pseudoaneurysms completely shrank. The aortic diameter at the injured level was exactly the same as the diameter of the aortic stent graft and thus exactly the same as the proximal and distal aortic necks.

\section{Midterm Results}

No deaths occurred during follow-up. No secondary endoleaks, no migrations, no twists, no kinks, and no device infections were observed during the follow-up phase. All the stent grafts were patent. Two $(6.1 \%)$ patients presented with a midterm complication. One patient presented with an asymptomatic thrombus at the distal part of the stent graft 2.9 years after the procedure, despite a daily low dose of aspirin. He was successfully treated with oral anticoagulation medication for 3 months (with an international normalized ratio of between 2 and 3). Another patient presented with an asymptomatic fracture of the nitinol stent (Talent; Medtronic, Inc) 7 years after the procedure.

Actuarial survival estimates and actuarial freedom from aortic reintervention 5 years after the procedure were $100 \%$. Actuarial freedom from complication 1 year after the procedure was $96.1 \% \pm 3.8 \%$, and it was $85.5 \% \pm 10.6 \%$ after 5 years.

\section{Discussion}

Since 1997 and the first reported endovascular procedure for blunt aortic rupture, more than 300 cases have been reported (as case reports or as limited series) with only a short follow-up time. Very little is known about the long-term results of such procedures. ${ }^{6,10-26}$ The purpose of this work, bearing on 33 consecutive patients with blunt isthmic ruptures that had been sustained at some point in the period from 1996 through 2005, is to report the midterm results of endovascular repair. After a mean follow-up of 2.7 years (with a maximum follow-up of 8 years in 1 patient), the overall survival rate is $100 \%$. The paraplegia rate is $0 \%$. No conversions to conventional surgical intervention and no endovascular reinterventions were required at the level of the aortic injury.

Actually, even the very best methods used in conventional surgical intervention for the repair of aortic ruptures of the descending thoracic aorta remain controversial. Despite technical advances, such as heparin-less partial cardiopulmonary bypass, extracorporeal circulation still in- 
duces a strong inflammatory response that can have deleterious consequences in the setting of an injurymediated inflammatory state. Furthermore, the mortality rate remains at $10 \%$ because any associated injuries might be worsened by the cardiopulmonary bypass. ${ }^{27}$ Although delayed conventional surgical intervention could be considered a good option in large trauma centers, and even if we did not observe any deaths during the waiting period, delayed surgical intervention is not completely risk free. A $4 \%$ risk of aortic rupture is reported despite the optimal management of patients in intensive care units. In addition, hypotensive therapy is challenging in cases of increased intracranial pressure, with a risk of a decrease in the cerebral perfusion pressure. ${ }^{5,28,29}$

At the beginning of our experience with the use of these procedures, delayed stent grafting was considered appropriate after recovery from associated life-threatening injuries for those patients with stable aortic lesions. ${ }^{6}$ Because the procedures were increasingly fast and without the need for thoracotomy, pulmonary exclusion, aortic crossclamping, and circulatory assistance, we observed lower perioperative morbidity and mortality rates than those observed after traditional surgical intervention, in spite of some patients in this trial having other associated life-threatening injuries (87.4\% of our patients had severe encephalic injuries). Because of the safety and simplicity of the procedures, like other authors, ${ }^{19,30}$ we decreased the delay period before treatment. Today, endovascular management can be performed in emergency situations at the price of an adapted organization in terms of the multidisciplinary team and the material available.

In spite of the attractive aspects of stent grafting, some technical difficulties have to be overcome. The often short proximal neck is the main difficulty. The ideal length of normal aorta upstream of the lesion of at least $15 \mathrm{~mm}$ is rarely encountered, and the ostium of the LSCA is very close to the aortic injury most of the time. Despite significant but relatively benign complications we encountered with the systematic use of the brachial route, we still use this approach to single out the ostium of the LSCA with a 5F 30-cm-long sheath with a distal radio-opaque marker. In our opinion this is crucial for optimal stent placement in the case of an isthmic lesion. In cases of intentional coverage of the LSCA, a mild systolic blood pressure differential was observed between the arms during the early postoperative phase, but all the patients remained asymptomatic. None of them complained about arm fatigue on exertion. Nevertheless, we can hypothesize that the overstenting of the LSCA might be a risk factor in the development of paraplegia because of the occlusion of the very proximal medullary artery and, more particularly, of the higher dorsal medullary artery. The only transient paraparesis we encountered occurred in a patient who had his LSCA occluded. The dom- inant or ectopic left vertebral artery is also to be considered carefully. From our point of view, this case would be an indication for the use of a carotidosubclavian bypass if the LSCA had to be covered. ${ }^{23}$ A preimplantation angiogram is actually mandatory to check the contralateral vertebral artery and the collaterals.

Today, the curvature of the distal aortic arch is more easily crossed with the new generation of more flexible stent grafts, but their size remains important. The vascular access is also still a potential technical problem in young patients with spastic arteries because a diameter of $6 \mathrm{~mm}$ at minimum is required to have a safe access. Infrainguinal iliofemoral access has always been possible, in our experience, but in cases in which this access is limited, suprainguinal iliac or infrarenal abdominal aortic accesses should be considered.

Last but not least, the size of the aorta in young patients, with diameters of less than $20 \mathrm{~mm}$, might be another limitation because of the lack of small, commercially available endovascular devices. From another point of view, these traumatisms sometimes occur in young patients whose aortas will increase in size.

As a whole, taking into account our excellent results in the short term and midterm, our management of blunt aortic ruptures has changed progressively. When aortic rupture is identified, 2 situations are singled out. In the case of aortarelated hemodynamic instability (massive mediastinal hematoma, active bleeding, or left hemothorax), emergency endovascular treatment is performed. In the case of nonaorta-related hemodynamic instability, the other lifethreatening injuries are first treated, and endovascular treatment of the aortic injury is performed within 24 hours. In the case of stable patients, endovascular management is performed within 24 hours if the patients present with contraindications to the use of conventional surgical management. The indications for surgical intervention are emergency operation in cases of collapse and massive left hemothorax preventing the carrying out of a CT scan and delayed surgical intervention in cases in which endovascular management is impossible for technical or anatomic reasons in patients with contraindications for emergency surgical intervention, including conventional emergency operations in the case of isolated isthmic rupture in young patients. A minor aortic injury, such as a limited intimal flap (defined as a less than 1-cm intimal flap with no or minimal periaortic hematoma) is observed and treated medically, with close clinical and radiologic follow-up.

\section{Conclusion}

This study, in spite of the limits related to its monocentric nonrandomized character, demonstrates that the endovascular treatment of blunt traumatisms of the descending thoracic aorta is a safe and effective therapeutic method with 
decreased midterm morbidity and mortality rates. Only controlled and randomized studies comparing conventional surgical intervention and endovascular repair would make it possible to confirm these results, but they would be difficult to realize because of the low number of patients treated in each center.

The main technical improvements should relate to the reduction in the size of the delivery devices and the use of more flexible devices. Stent grafts with a "proximal subclavian branch" would also make it possible to avoid the sacrifice of the LSCA and would allow a more satisfactory proximal sealing.

In spite of these very good results in the short term and midterm, the long-term results of endovascular repair remain unknown. It is also essential to continue close morphologic monitoring. In the case of hypothetic long-term complications, endovascular procedures would be possible, and if conventional surgical intervention was necessary, the operation would be performed with hemodynamic stability and thus under much better conditions than when being undertaken in an emergency.

We acknowledge the kind help of Mrs Barbara Hollington for the preparation of the revised version of this manuscript.

\section{References}

1. Jahromi AS, Kazemi K, Safar HA, et al. Traumatic rupture of the thoracic aorta: cohort study and systematic review. J Vasc Surg. 2001;34:1029-34.

2. Cowley RA, Turney SZ, Hankins JR, et al. Rupture of the thoracic aorta caused by blunt trauma, a fifteen-year experience. $J$ Thorac Cardiovasc Surg. 1990;100:652-60.

3. von Oppell UO, Dunne TT, DeGroot MK, et al. Traumatic aortic rupture: twenty-year metananalysis of mortality and risk of paraplegia. Ann Thorac Surg. 1994;58:585-93.

4. Fabian TC, Richardson JD, Croce MA, et al. Prospective study of blunt aortic injury: multicenter trial of the American Association for the Surgery of Trauma. J Trauma. 1997;42:374-80.

5. Pate JW, Fabian TC, Walker W. Traumatic rupture of the aortic isthmus: an emergency? World J Surg. 1995;19:152-99.

6. Rousseau H, Soula P, Perreault P, et al. Delayed treatment of traumatic rupture of the thoracic aorta with endoluminal covered stent. Circulation. 1999;99:498-504.

7. Chaikof EL, Blankensteijn JD, Harris PL, et al. Reporting standards for endovascular aortic aneurysm repair. J Vasc Surg. 2002;35:104860.

8. White GH, Yu W, May J, et al. Endoleak as a complication of endoluminal grafting of abdominal aortic aneurysms: classification, incidence, diagnosis and management. J Endovasc Surg. 1997;4:15268.

9. White GH, May J, Waugh RC, et al. Type III and type IV endoleak: toward a complete definition of blood flow in the sac after endoluminal AAA repair. J Endovasc Surg. 1998;5:305-9.
10. Doss M, Balzer J, Martens S, et al. Surgical versus endovascular treatment of acute thoracic aortic rupture: a single-center experience. Ann Thorac Surg. 2003;76:1465-9.

11. Nienaber CA, Ince H, Weber F, et al. Emergency stent-graft placement in thoracic aortic dissection and evolving rupture. J Card Surg. 2003; 18:464-70.

12. Orend KH, Pamler R, Kapfer X, et al. Endovascular repair of traumatic descending aortic transection. J Endovasc Ther. 2002;9:573-8.

13. Rousseau H, Dambrin C, Marcheix B, et al. Acute traumatic aortic rupture: a comparison of surgical and stent-graft repair. J Thorac Cardiovasc Surg. 2005;129:1050-5.

14. Kato N, Dake MD, Miller DC, et al. Traumatic thoracic aortic aneurysm: treatment with endovascular stent-grafts. Radiology. 1997;205: $657-62$.

15. Semba CP, Kato N, Kee ST, et al. Acute rupture of the descending thoracic aorta: repair with use of endovascular stent-grafts. J Vasc Interv Radiol. 1997;8:337-42.

16. Fujikawa T, Yukioka T, Ishimaru S, et al. Endovascular stent grafting for the treatment of blunt thoracic aortic injury. J Trauma. 2001;50: 223-9.

17. Fattori R, Napoli G, Lovato L, et al. Indications for, timing of, and results of catheter-based treatment of traumatic injury to the aorta. AJR Am J Roentgenol. 2002;179:603-9.

18. Hoffer EK, Karmy-Jones R, Bloch RD, et al. Treatment of acute thoracic aortic injury with commercially available abdominal aortic stent-grafts. J Vasc Interv Radiol. 2002;13:1037-41.

19. Lachat M, Pfammatter T, Witzke H, et al. Acute traumatic aortic rupture: early stent-graft repair. Eur J Cardiothorac Surg. 2002;21: 959-63.

20. Thompson CS, Gaxotte VD, Rodriguez JA, et al. Endoluminal stent graft repair of aortobronchial fistulas. J Vasc Surg. 2002;35:387-91.

21. Orford VP, Atkinson NR, Thomson K, et al. Blunt traumatic aortic transection. Ann Thorac Surg. 2003;75:100-11.

22. Bortone AS, De Cillis E, D'Agostino D, et al. Endovascular treatment of thoracic aortic disease: four years of experience. Circulation. 2004; 110(suppl):II262-7.

23. Dunham MB, Zygun D, Petrasek P, et al. Endovascular stent grafts for acute blunt aortic injury. J Trauma. 2004;56:1173-8.

24. Iannelli G, Piscione F, Di Tommaso L, et al. Thoracic aortic emergencies: impact of endovascular surgery. Ann Thorac Surg. 2004;77: 591-6.

25. Melnitchouk S, Pfammatter T, Kadner A, et al. Emergency stent-graft placement for hemorrhage control in acute thoracic aortic rupture. Eur J Cardiothorac Surg. 2004;25:1032-8.

26. Rousseau H, Dambrin C, Marcheix B, et al. Acute traumatic aortic rupture: a comparison of surgical and stent-graft repair. J Thorac Cardiovasc Surg. 2005;129:1050-5.

27. Downing SW, Cardarelli MG, Sperling J, et al. Heparinless partial cardiopulmonary bypass for the repair of aortic trauma. $J$ Thorac Cardiovasc Surg. 2000;120:1104-11.

28. Holmes JH, Bloch RD, Hall A, et al. Natural history of traumatic rupture of the thoracic aorta managed nonoperatively: a longitudinal analysis. Ann Thorac Surg. 2002;73:1149-54.

29. Cook J, Salerno C, Krishnadasan B, et al. The effect of changing presentation and management on the outcome of blunt rupture of the thoracic aorta. J Thorac Cardiovasc Surg. 2006;131:594-600.

30. Ahn SH, Cutry A, Murphy TP, et al. Traumatic thoracic aortic rupture: treatment with endovascular graft in the acute setting. J Trauma. 2001;50:949-51. 